\title{
CMOS Compatible Silicon-on-Insulator Polarization Rotator Based on Symmetry Breaking of the Waveguide Cross Section
}

\author{
Mariam Aamer, Ana M. Gutierrez, Antoine Brimont, Diedrik Vermeulen, Gunther Roelkens, \\ Jean-Marc Fedeli, Andreas Håkansson, and Pablo Sanchis
}

\begin{abstract}
A polarization rotator in silicon-on-insulator technology based on breaking the symmetry of the waveguide cross section is reported. The $25-\mu \mathrm{m}$-long device is designed to be integrated with standard grating couplers without the need for extra fabrication steps. Hence, fabrication is carried out by a 2-etch-step complementary metal-oxide-semiconductor compatible process using 193-nm deep ultraviolet lithography. A polarization conversion efficiency of more than $-0.85 \mathrm{~dB}$ with insertion losses ranging from -1 to $-2.5 \mathrm{~dB}$ over a wavelength range of $30 \mathrm{~nm}$ is demonstrated.
\end{abstract}

Index Terms - Integrated optics, polarization rotator, siliconon-insulator (SOI).

\section{INTRODUCTION}

$\mathbf{S}$ ILICON-ON-INSULATOR (SOI) is being consolidated as the most promising technology to develop photonic devices for telecom applications with an ultra large scale of integration (ULSI) at a low cost [1] due to the high refractive index contrast between silicon $(\mathrm{n} \approx 3.45)$ and silica $(\mathrm{n} \approx 1.45)$ at $\lambda=1.55 \mu \mathrm{m}$, as well as its compatibility with CMOS microelectronic tools.

For high index-contrast integrated waveguides, one of the main issues is their high birefringence. To overcome this drawback, a polarization diversity scheme is required for enabling polarization-insensitivity to arbitrary polarized input light, with the polarization rotator being one of the core devices [2]. Several approaches have been proposed to rotate the polarization in silicon waveguides. Mode evolution based rotation

Manuscript received July 11, 2012; revised August 27, 2012; accepted September 8, 2012. Date of publication September 12, 2012; date of current version October 31, 2012. This work was supported by the European Commission under Project HELIOS (pHotonics Electronics functional Integration on CMOS), FP7-224312, TEC2008-06333 SINADEC and PROMETEO-2010087 R\&D Excellency Program (NANOMET).

M. Aamer, A. M. Gutierrez, A. Brimont, and P. Sanchis are with the Nanophotonics Technology Center, Universitat Politècnica València, València 46022, Spain (e-mail: maaa@ntc.upv.es; angucam@ntc.upv.es; abrimont@ntc.upv.es; pabsanki@ntc.upv.es).

D. Vermeulen and G. Roelkens are with the Department of Information Technology, Photonics Research Group, Ghent University-IMEC, Ghent B-9000, Belgium (e-mail: diedrik.vermeulen@intec.ugent.be; gunther.roelkens@intec.ugent.be).

J.-M. Fedeli is with the Commissariat á l'Energie Atomique, Laboratoire d'Électronique des Technologies de l'Information, MINATEC Institute, Grenoble 38054, France (e-mail: jean-marc.fedeli@cea.fr).

A. Håkansson is with DAS Photonics S.L., Ciudad Politécnica de la Innovación, València 46022, Spain (e-mail: ahakansson@dasphotonics.com). has been demonstrated by twisting the waveguide using two asymmetric layers oppositely tapered [3]. However, in this case the input and output silicon waveguides have different thicknesses imposing a serious restriction when integrated in a more complex device. Alternatively, an asymmetrical directional coupler has been demonstrated which only requires one lithography and etching step [4] and that can also be used for splitting the polarization [5], [6]. However, longer lengths are required and the vertical symmetry of the coupler must also be broken by using a different top-cladding material from that of the buffer layer which again would impose restrictions for its integration. In this way, the use of different materials on top of single silicon waveguides have also been demonstrated for achieving polarization rotation [7], [8].

A pure silicon solution without the need of extra materials can be achieved by breaking the symmetry of the waveguide cross section. Hence, the propagation modes are hybridized allowing power to be transferred periodically between the two desired polarization states. Initially, angled waveguides were demonstrated but requiring complicated fabrication processes [9]. Therefore, a simple two-step etching to build a stair-like cross section was proposed which in addition shortened the rotation length [10]. Multiple subwavelength trenches [11] or adding a polycrystalline silicon overlay [12] have been proposed and demonstrated to minimize the rotation lengths but once more at the expense of increasing the number of abrication steps.

In this letter, a simple and compact two-step etching based polarization rotator following the original idea from [10] is designed and demonstrated using a CMOS compatible fabrication process with $193 \mathrm{~nm}$ deep ultraviolet (UV) lithography. The design is especially carried out to be compatible with standard SOI wafers with $220 \mathrm{~nm}$ thick silicon layer and its associated standard grating couplers for eff cient coupling to external optical fiber [13].

\section{Polarization Rotator Structure}

The proposed polarization rotator consists of a straight asymmetric structure shallow etched in a strip waveguide conf guration as depicted in the inset of Fig. 1. The chosen parameters are $h=220 \mathrm{~nm}$ (silicon thickness in standard SOI wafers), etch depth, ed $=70 \mathrm{~nm}$, and $\mathrm{f} 11$ factor, $f f$, of $25 \%$. The etch depth was chosen to facilitate the integration of the 


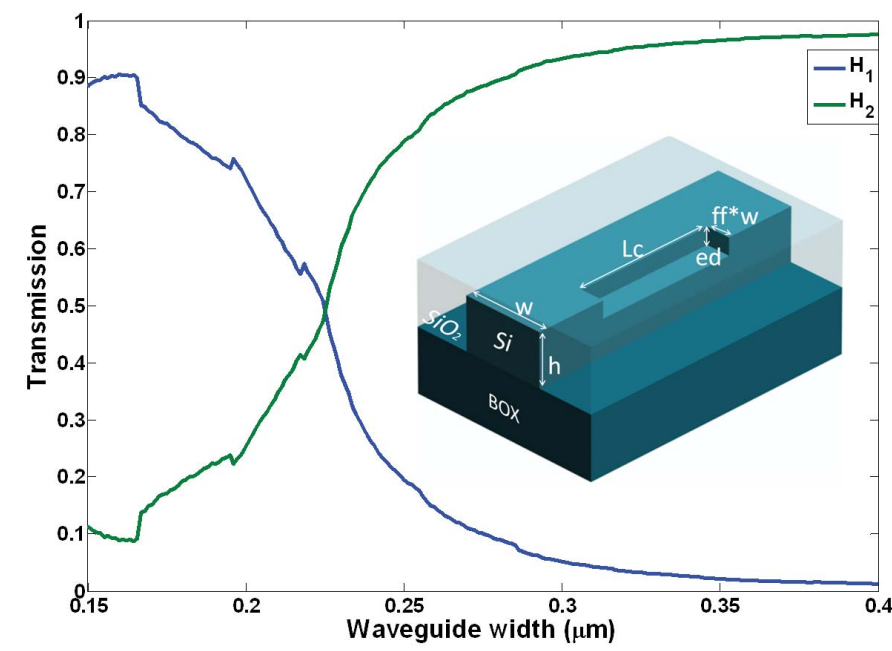

Fig. 1. Simulated excitation of hybrid modes as a function of the waveguide width of the proposed polarization rotator shown in the inset, at the symmetricasymmetric interface.

polarization rotator in devices that use conventional gratings to couple light from an external optical f ber [13]. A symmetricasymmetric waveguide interface allows the excitation of two hybrid modes $H_{1}$ and $H_{2}$ with their principal axis tilted by 45 degrees. In order to achieve total polarization conversion, these hybrid modes must be excited equally. This condition is satisf ed for a waveguide width of around $220 \mathrm{~nm}$, as shown in Fig. 1.

The polarization rotator was then simulated and analyzed with a fully vectorial and bi-directional optical propagation tool based on eigenmode expansion [14]. Fig. 2 shows the polarization conversion eff ciency (PCE) as a function of the rotator length, where it can be concluded that for the optimal theoretical length value $(\sim 23 \mu \mathrm{m})$, the PCE is almost perfect.

\section{FABRICATION PROCESS}

The complete CMOS fabrication process was carried out by using $193 \mathrm{~nm}$ deep UV lithography. It starts with $100 \mathrm{~nm}$ high temperature oxide (HTO) on top of a SOITEC wafer consisting of a $220 \mathrm{~nm}$ thick silicon epilayer on top of a $2 \mu \mathrm{m}$ thick BOX. The gratings and the waveguide arms are $\mathrm{f} \mathrm{rst}$ patterned, followed by reactive ion etching (RIE) silica with $\mathrm{C}_{4} \mathrm{~F}_{8}$, which defi es a hardmask. The silicon is then partially etched with $\mathrm{HBr}$ and controlled by ellipsometry in order to defi e precisely the grating teeth and the polarization rotator etch depth. In the second lithography step, the gratings and the polarization rotator are protected by the resist and the remaining hardmask serves for the waveguides in a self-alignment process. Then a full silicon etch down to the buffer silica layer completes the waveguide fabrication. Finally, a top silica cladding was deposited achieving around $1 \mu \mathrm{m}$ thickness.

Fig. 3 shows a schematic of the whole polarization diversity structure used to characterize the polarization rotator, with scanning electron microscope (SEM) images of the different parts of the circuit. As it can be seen, the fir t element is a TE focusing grating coupler with a period of $630 \mathrm{~nm}$ and a coupling eff ciency of around $-5 \mathrm{~dB}$. Fig. 3(b) shows a

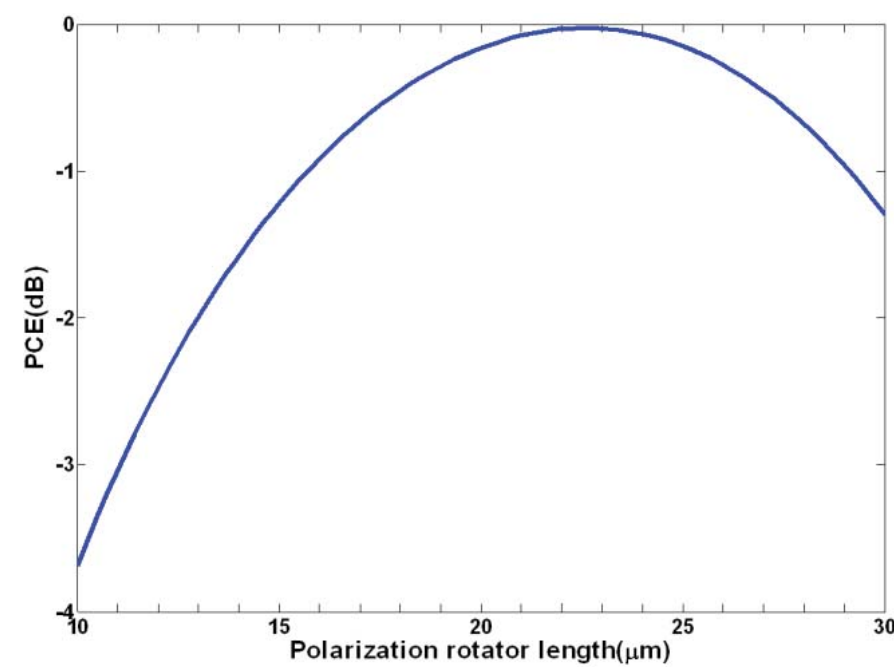

Fig. 2. PCE as a function of the polarization rotator length for the optimum waveguide width of $220 \mathrm{~nm}$.

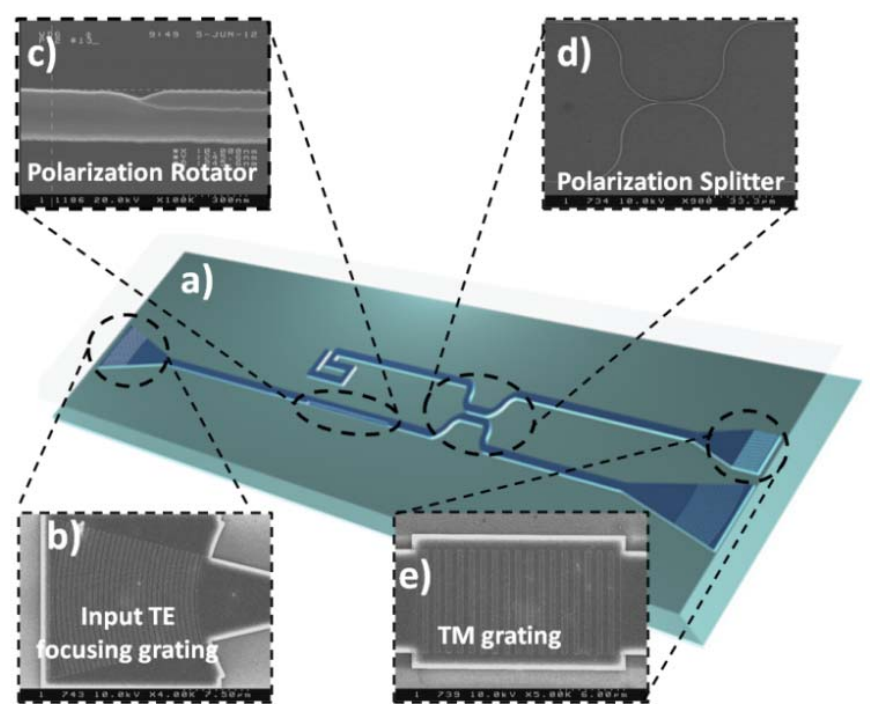

Fig. 3. (a) Schematic of the whole polarization diversity structure used to characterize the polarization rotator. SEM images of (b) TE input focusing grating coupler, (c) polarization rotator, (d) polarization splitter, and (e) TM output grating coupler.

SEM image of the TE focusing grating. The next structure the TE polarized light propagates along is the polarization rotator, with the parameters previously designed. A top SEM image of the fabricated rotator is depicted in Fig. 3(c). At this point, the TE and TM modes are separated by a $13 \mu \mathrm{m}$-length directional coupler, shown in Fig. 3(d), with a gap of $300 \mathrm{~nm}$, acting as a polarization splitter with a measured extinction ratio above $25 \mathrm{~dB}$. Once the two polarizations are separated, the TE and TM modes travel along the splitter output arms, to be recollected by the output fber, through the TE and TM output grating couplers, respectively. The TM output grating, with a period of $1 \mu \mathrm{m}$, is depicted in Fig. 3(e), and has a coupling eff ciency of around $-6 \mathrm{~dB}$.

\section{EXPERIMENTAL Results}

Polarization rotators with different lengths were fabricated and characterized. Measurements were performed with a single 


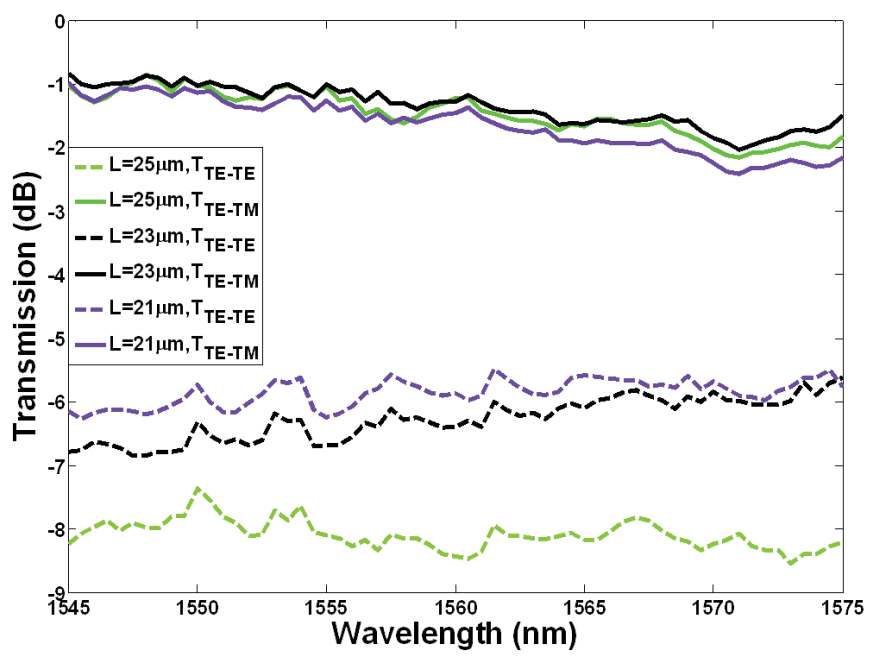

Fig. 4. Normalized measured spectra as a function of wavelength of polarization rotators with three different lengths.

mode fibe tilted under an angle of 13 degrees relative to the surface normal of the grating. The input polarization state was f rstly set by adjusting an external polarization controller and monitoring the output power measured in a reference straight waveguide. The TE-TM coupling eff ciency, which is the key characteristic of the proposed device, has to be maximized for optimum performance. However, because of reciprocity, the polarization rotation from TE to TM will have the same efficie cy as the rotation from TM to TE. Therefore, the polarization conversion eff ciency, which is a measure of the extinction ratio at the output port, has been defi ed as

$$
P C E(d B)=10 \log _{10}\left(\frac{T_{T E-T M}}{T_{T E-T E}+T_{T E-T M}}\right)
$$

where $\mathrm{T}_{\mathrm{TE}-\mathrm{TM}}$ is the power coupled to $\mathrm{TM}$ polarization when the input polarization is $\mathrm{TE}$ while $\mathrm{T}_{\mathrm{TE}-\mathrm{TE}}$ is the power of the undesired non-rotated TE polarization for the same case. The eff ciency of the rotator was thus measured by comparing the TE and TM polarization after coupling a TE polarization to the circuit, without any residual TM polarization due to the fltering effect produced by the input grating coupler, then passing through the rotator and f nally using the polarization splitter to separate the rotated TM mode from the undesired non-rotated TE one.

Fig. 4 shows the normalized measured spectra of the polarization diversity structure shown in Fig. 3 at the two outputs, namely $\mathrm{T}_{\mathrm{TE}-\mathrm{TM}}$ and $\mathrm{T}_{\mathrm{TE}-\mathrm{TE}}$, for three different lengths of the polarization rotator. The normalization was carried out taking into account the input and output gratings as well as the polarization splitter. As it can be seen, the insertion loss is ranging from $-1 \mathrm{~dB}$ to $-2.5 \mathrm{~dB}$ for the different characterized devices. Fig. 5 shows the PCE as a function of the wavelength, again for the three different rotator lengths. As the rotator length moves away from the optimal one, the TE-TE coupling eff ciency increases, as plotted in Fig. 4 (dashed lines), whereas the change in the TE-TM coupling (solid lines) is much lower, according with the theoretical behavior. The measured PCE is above $-0.85 \mathrm{~dB}$ over a wavelength range of $30 \mathrm{~nm}$, and it is achieved for the

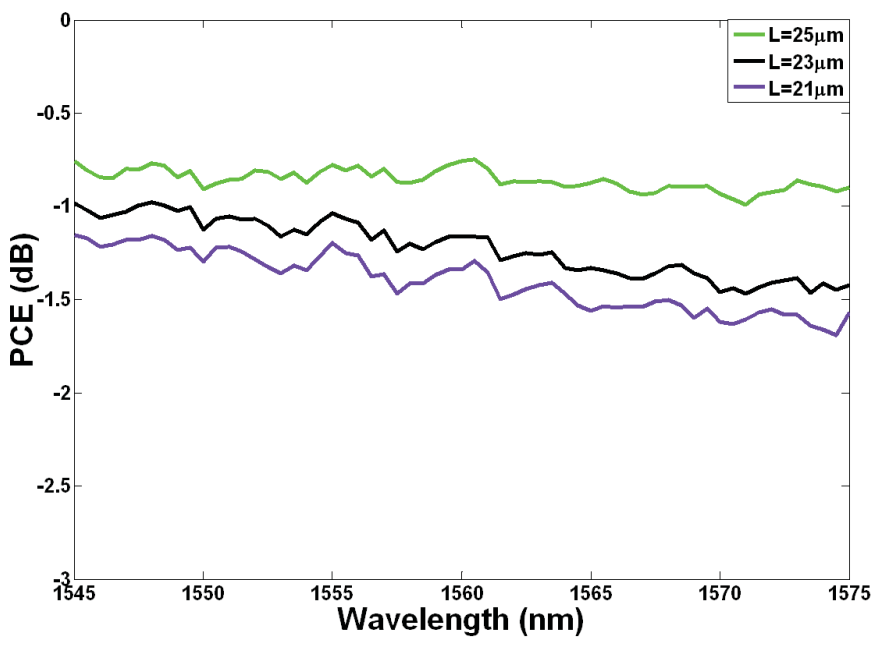

Fig. 5. PCE as a function of wavelength for the different lengths of the polarization rotators.

polarization rotator which length is $25 \mu \mathrm{m}$. When results shown in Fig. 2 and Fig. 5 are compared, it can be seen that there is a very good agreement between the simulated and measured variation of the PCE with the rotator length. Small discrepancies are mainly originated due to deviations between the target and fabricated parameters of the rotator. However, it should be pointed out that the rotator parameters may change in the range of a few tens of nanometers without decreasing the PCE in more than $0.5 \mathrm{~dB}$ [10], [12]. Therefore, it can be stated that the robustness of the proposed polarization rotator device meets CMOS fabrication tolerances based on $193 \mathrm{~nm}$ deep UV lithography.

\section{CONCLUSION}

In conclusion, a fully CMOS compatible compact and broadband polarization rotator in SOI technology has been designed, fabricated and experimentally characterized. The $25 \mu \mathrm{m}$-long-device features a polarization conversion eff ciency above $-0.85 \mathrm{~dB}$ over a wavelength range of $30 \mathrm{~nm}$ with insertion loss ranging from $-1 \mathrm{~dB}$ to $-2.5 \mathrm{~dB}$.

\section{REFERENCES}

[1] B. Jalali and S. Fathpour, "Silicon photonics," J. Lightw. Technol., vol. 24, no. 12, pp. 4600-4615, Dec. 2006.

[2] T. Barwicz, et al., "Polarization-transparent microphotonic devices in the strong confine ent limit," Nature Photon., vol. 1, pp. 57-60, Dec. 2006.

[3] J. Zhang, M. Yu, G. Lo, and D. Kwong, "Silicon-waveguide-based mode evolution polarization rotator," IEEE J. Sel. Topics Quantum Electron., vol. 16, no. 1, pp. 53-60, Jan./Feb. 2010.

[4] L. Liu, Y. Ding, K. Yvind, and J. M. Hvam, "Eff cient and compact TE-TM polarization converter built on silicon-on-insulator platform with a simple fabrication process," Opt. Lett., vol. 36, no. 7, pp. 1059-1061, 2011.

[5] D. Dai and J. E. Bowers, "Novel concept for ultracompact polarization splitter-rotator based on silicon nanowires," Opt. Express, vol. 19, no. 11, pp. 10940-10949, 2011.

[6] L. Liu, Y. Ding, K. Yvind, and J. M. Hvam, "Silicon-on-insulator polarization splitting and rotating device for polarization diversity circuits," Opt. Express, vol. 19, no. 13, pp. 12646-12651, 2011.

[7] H. Fukuda, K. Yamada, T. Tsuchizawa, T. Watanabe, H. Shinojima, and S. Itabashi, "Polarization rotator based on silicon wire waveguides," Opt. Express, vol. 16, no. 4, pp. 2628-2635, 2008. 
[8] L. Chen, C. R. Doerr, and Y. Chen, "Compact polarization rotator on silicon for polarization-diversif ed circuits," Opt. Lett., vol. 36, no. 4, pp. 469-471, 2011.

[9] C. Brooks, P. E. Jessop, H. Deng, D. O. Yevick, and G. Tarr, "Passive silicon-on-insulator polarization-rotating waveguides," Opt. Eng., vol. 45, no. 4, p. 044603, Apr. 2006.

[10] Z. Wang and D. Dai, "Ultrasmall Si-nanowire-based polarization rotator," J. Opt. Soc. Amer. B, Opt. Phys., vol. 25, no. 5, pp. 747-753, 2008.

[11] A. V. Velasco, et al., "Ultracompact polarization converter with a dual subwavelength trench built in a silicon-on-insulator waveguide," Opt. Lett., vol. 37, no. 3, pp. 365-367, 2012.
[12] D. Vermeulen, et al., "Silicon-on-insulator polarization rotator based on a symmetry breaking silicon overlay," IEEE Photon. Technol. Lett., vol. 24, no. 6, pp. 482-484, Mar. 15, 2012.

[13] D. Taillaert, et al., "An out-of-plane grating coupler for eff cient butt-coupling between compact planar waveguides and single-mode fib rs," IEEE J. Quantum Electron., vol. 38, no. 7, pp. 949-955, Jul. 2002.

[14] Commercial Software by Photon Design. (2010) [Online]. Available: http://www.photond.com 